\title{
Novel cell separation method for molecular analysis of neuron-astrocyte co-cultures
}

\section{Andrea Goudriaan ${ }^{1 \dagger}$, Nutabi Camargo $^{1+}{ }^{\dagger}$, Karen E. Carney ${ }^{1,2,3}{ }^{\dagger}$, Stéphane H. R. Oliet ${ }^{2,3}$, August B. Smit $^{1}$ and Mark H. G. Verheijen ${ }^{1 *}$}

' Department of Molecular and Cellular Neurobiology, Center for Neurogenomics and Cognitive Research, Neuroscience Campus Amsterdam, VU University Amsterdam, Amsterdam, Netherlands

2 INSERM U862, Neurocentre Magendie, Bordeaux, France

${ }^{3}$ Université de Bordeaux, Bordeaux, France

\section{Edited by:}

Martin Stangel, Hannover Medical School, Germany

\section{Reviewed by:}

Flavia Carvalho Alcantara Gomes, Federal University of Rio de Janeiro, Brazil

Frank W. Pfrieger, Centre National de la Recherche Scientifique, France

\section{${ }^{*}$ Correspondence:}

Mark H. G. Verheijen, Department of Molecular and Cellular Neurobiology Center for Neurogenomics and Cognitive Research, Neuroscience Campus Amsterdam, VU University Amsterdam, Boelelaan 1085, 1081 HV Amsterdam, Netherlands e-mail:mark.verheijen@vu.nI

${ }^{\dagger}$ Andrea Goudriaan, Nutabi Camargo, and Karen E. Carney have contributed equally to this work.
Over the last decade, the importance of astrocyte-neuron communication in neuronal development and synaptic plasticity has become increasingly clear. Since neuron-astrocyte interactions represent highly dynamic and reciprocal processes, we hypothesized that many astrocyte genes may be regulated as a consequence of their interactions with maturing neurons. In order to identify such neuron-responsive astrocyte genes in vitro, we sought to establish an expedited technique for separation of neurons from co-cultured astrocytes. Our newly established method makes use of cold jet, which exploits different adhesion characteristics of subpopulations of cells (Jirsova etal., 1997), and is rapid, performed under ice-cold conditions and avoids protease-mediated isolation of astrocytes or time-consuming centrifugation, yielding intact astrocyte mRNA with approximately $90 \%$ of neuronal RNA removed. Using this purification method, we executed genome-wide profiling in which RNA derived from astrocyte-only cultures was compared with astrocyte RNA derived from differentiating neuron-astrocyte co-cultures. Data analysis determined that many astrocytic mRNAs and biological processes are regulated by neuronal interaction. Our results validate the cold jet as an efficient method to separate astrocytes from neurons in co-culture, and reveals that neurons induce robust gene-expression changes in co-cultured astrocytes.

Keywords: method, co-culture, astrocytes, transcriptional activation, isolation approaches, neuron-glia interaction

\section{INTRODUCTION}

During central nervous system (CNS) development, glia and neurons are targeted to their proper location in the brain and the appropriate contacts between neurons, as well as neurons and glia cells, are established. While neuroscience research historically has focused on synaptic contacts between neurons, the last couple of decades it has become apparent that reciprocal neuron-glia interactions are crucial for correct brain functioning. The functionally diverse glia-neuron interactions rely on a range of both contact-dependent mechanisms as well as soluble factors (Eroglu and Barres, 2010; Giaume etal., 2010). The development of elaborate in vitro systems, using highly purified cell populations, has strongly contributed to the elucidation of the molecular effects of glia on neuronal development and function (Ullian et al., 2001; Thomson et al., 2008; Jarjour et al., 2012). Specifically, the use of astrocyte-neuron co-cultures has led to the identification of several astrocyte-derived signals that induce neuritogenesis and synaptogenesis, at least in vitro. For instance, lipids are secreted by astrocytes in high quantities and are well known to promote neurite outgrowth and synapse formation, (Mauch et al., 2001; Medina and Tabernero, 2002; Goritz et al., 2005; Camargo et al., 2009; Verheijen et al., 2009). Other astrocyte-secreted factors that were shown to influence synapse development are extracellular matrix molecules, such as thrombospondins and glypicans (Clarke and Barres, 2013).

Whereas new astrocyte factors affecting neuronal function are likely to be discovered, a prominent deficit in our knowledge is how expression and secretion of these astrocyte factors are controlled. Neuronal synaptic activity has been shown to induce transcriptional activity in astrocytes (Mendez et al., 2004). It thus follows that synaptic activity and neuron-glia communication will likely elicit gene expression changes in glia, which in turn might reciprocally affect the process of synapse maturation and synaptic transmission. In order to identify neuron-responsive astrocyte genes, we established a developmental in vitro transcriptional profiling experiment using neuron-astrocyte co-cultures. To specifically isolate astrocytes from the co-culture, we successfully developed a fast method, named "cold jet" that exploits different adhesion characteristics of subpopulations of cells (Jirsova et al., 1997). The method lacks disadvantages of other methods, e.g., that of fluorescence activated cell sorting (FACS) or magnetic bead-based cell sorting involving protease-mediated dissociation of cells (Lovatt etal., 2007; Foo, 2013), which cannot be efficiently conducted at low temperatures, thereby compromising both protein and mRNA quality. Using cold jet-mediated isolation of astrocytes, followed by microarray analysis, we identified nearly 400 mRNAs significantly regulated in astrocytes when co-cultured 
with neurons. The resulting database confirms the validity of the used cold jet method for separation of astrocytes and neurons, and represents an important gene source toward understanding of neuron-glia communication.

\section{METHODS}

\section{CELL CULTURE}

Neuron-astrocyte co-cultures were done using rat astrocytes, because of tissue culture advantages of rat over mouse astrocytes, and mouse neurons to make it possible for future experiments to use neurons of genetically modified mice. Importantly, in these co-cultures, rat astrocyte provide the required support for optimal synaptic functioning of co-cultured mouse neurons (Wierda et al., 2007), an important requirement for our screen of neuron-induced expression changes in astrocytes. Primary rat astrocytes were obtained from Wistar rat pups (P1). Briefly, dissected cortices were treated with trypsin (Gibco) followed by addition of trypsin inhibitor and gentle trituration, after which cells were resuspended in Dulbecco's modified Eagle's medium (DMEM) + Glutamax (Gibco) containing 10\% fetal bovine serum and cultured in non-coated plastic flasks. Medium was changed every 2 days. The purity of astrocyte cultures was determined by immunostaining for glial fibrillary acidic protein (GFAP) and S100 $\beta$, while counterstained for all cell nuclei using Hoechst, and was determined to be higher than $98 \%$. For neuron-astrocyte cocultures, astrocytes were plated at a density of $30 \mathrm{~K} /$ well in 12 -wells plates in DMEM $+10 \%$ medium, which resulted in an about $80 \%$ confluent monolayer at day in vitro (DIV) 3, after which neurons were plated. For co-plating of neurons, hippocampi of E18 mouse embryos were collected in Hanks buffered salts solution (HBSS; Sigma), buffered with 7 mM 4-(2-hydroxyethyl)1-piperazineethanesulfonic acid (HEPES). After removal of the meninges, hippocampi were minced and incubated for $20 \mathrm{~min}$ in $0.25 \%$ trypsin in HBSS at $37^{\circ} \mathrm{C}$. After washing, the neurons were triturated with fire-polished Pasteur pipettes, counted, and plated in culture medium [Neurobasal medium (Invitrogen, Carlsbad, USA) supplemented with 2\% B-27 (Invitrogen), $1.8 \%$ HEPES, $1 \%$ glutamax (Invitrogen), 1\% Pen/Strep (Invitrogen), and $0.2 \%$ beta-mercaptoethanol] at a density of $60 \mathrm{~K} /$ well onto the astrocyte monolayers in 12-well plates $(4 \mathrm{~cm} \times 4 \mathrm{~cm}$; Greiner bio-one; Wierda et al., 2007). Wells were coated with $20 \%$ d-poly-lysine (Sigma) and 20\% collagen diluted in a $17 \mu \mathrm{M}$ acetic acid $\mathrm{H}_{2} \mathrm{O}$ solution. Cultures were maintained in $5 \% \mathrm{CO}_{2}$ at $37^{\circ} \mathrm{C}$, and half of the medium was changed at 1 day and 7 days after plating. Control conditions (astrocytes alone) were kept under similar tissue culture conditions as neuron-astrocyte co-cultures.

\section{COLD JET SEPARATION METHOD}

For removal of neurons from the co-cultured astrocytes, we modified a method previously used for removal of Schwann cells from co-cultured fibroblasts (Jirsova et al., 1997). 12-well tissue culture plates were placed on ice and the medium was gently aspirated. The cells were carefully rinsed once with ice-cold phosphate buffered saline (PBS; pH 7.4). Next, using a $1 \mathrm{~mL}$ pipette tip, $1 \mathrm{~mL}$ of ice-cold PBS was pipetted onto the co-cultures using a concentric circular motion, ensuring the force of the stream contacted the entire well surface area, which was repeated three times. This resulted in specific detachment of neurons, which were aspirated, leaving purified astrocytes, which were harvested for molecular analysis. All together, the isolation of astrocytes from co-cultures for molecular isolation per well was performed within a timeframe of $30 \mathrm{~s}$. When co-culture cold jet (CC-CJ) cultures were compared to astrocyte alone cultures, the later were also treated by cold jet, therefore called astrocyte-alone cold jet (AA-CJ) samples.

\section{IMMUNOCYTOCHEMISTRY}

Astrocyte monolayers or astrocyte-neuron co-cultures, before or after cold jet, were fixed by $4 \%$ paraformaldehyde in $0.1 \mathrm{M}$ PBS for $15 \mathrm{~min}$ at room temperature (RT). Cells were incubated $30 \mathrm{~min}$ in $0.1 \mathrm{M}$ PBS containing $0.5 \%$ TritonX-100 and $10 \%$ normal goat serum, and subsequently incubated with appropriate primary antibody concentrations in incubating medium (0.1 M PBS, $0.1 \%$ TritonX-100, $2 \%$ normal goat serum) at $4{ }^{\circ} \mathrm{C}$ overnight. Cells were washed three times for $10 \mathrm{~min}$ in $0.1 \mathrm{M}$ PBS and incubated in incubating medium containing the appropriated secondary antibody for $2 \mathrm{~h}$. Sections were washed two times with 0.1 M PBS, one time with water, and subsequently DNA counterstained in Hoechst reagent 33258 solution (Sigma). The primary antibodies were rabbit anti-GFAP (1:500; Dako) and chicken anti-microtubule-associated protein 2 (MAP2; 1:5000; Abcam). Secondary antibodies were donkey anti-rabbit Cy3 (1:400; Jackson laboratories), goat anti-chick Alexa 488 (1:400; Molecular probes).

\section{RNA ISOLATION, REVERSE TRANSCRIPTION, AND QUANTITATIVE PCR}

Total RNA was extracted from 12-well plate and mRNA was isolated with Trizol Reagent (Invitrogen). RNA samples for microarray analysis were derived from two to four tissue culture wells, and microarray replicates were derived from different pools of animals, with five to eight animals per pool. An additional chloroform extraction was performed to remove traces of phenol and samples were suspended in $\mathrm{H}_{2} \mathrm{O}$. The quality and quantity of RNA samples for microarray analysis were assessed on Agilent RNA chips with the Agilent 2100 Bioanalyzer (Agilent Technologies). For quantitative PCR, four samples were used that were different than those used for microarray analysis, and each sample was derived from different animals and four to six tissue culture wells. RNA integrity was determined using electrophoresis with RNA loading dye on a $1 \%$ agarose gel, RNA quantity was determined by photospectrometry (ND-1000; Thermo Scientific). Quantitative PCR reactions were done as described (Camargo et al., 2012).

\section{MICROARRAY ANALYSIS}

Agilent whole rat genome $4 \mathrm{x} 44 \mathrm{~K}$ chips were used. cDNA synthesis and labeling for microarray hybridization were performed according to the manufacturers' instructions. Briefly, oligo dT primers were used and Cy3 and Cy5 labeling was done using the Agilent two-color RNA Spike-In Kit. $0.5 \mu \mathrm{g}$ of Cy5 and Cy3-labeled cRNA targets were hydrolyzed and mixed for $30 \mathrm{~min}$. Arrays were hybridized with Cy5 and Cy3 solution for $18 \mathrm{~h}$ at $60^{\circ} \mathrm{C}$ in a rotating hybridization chamber, using $1 \times$ hybridization solution (Agilent technologies). Subsequently, arrays were washed, spun dry and scanned using an Agilent scanner. 

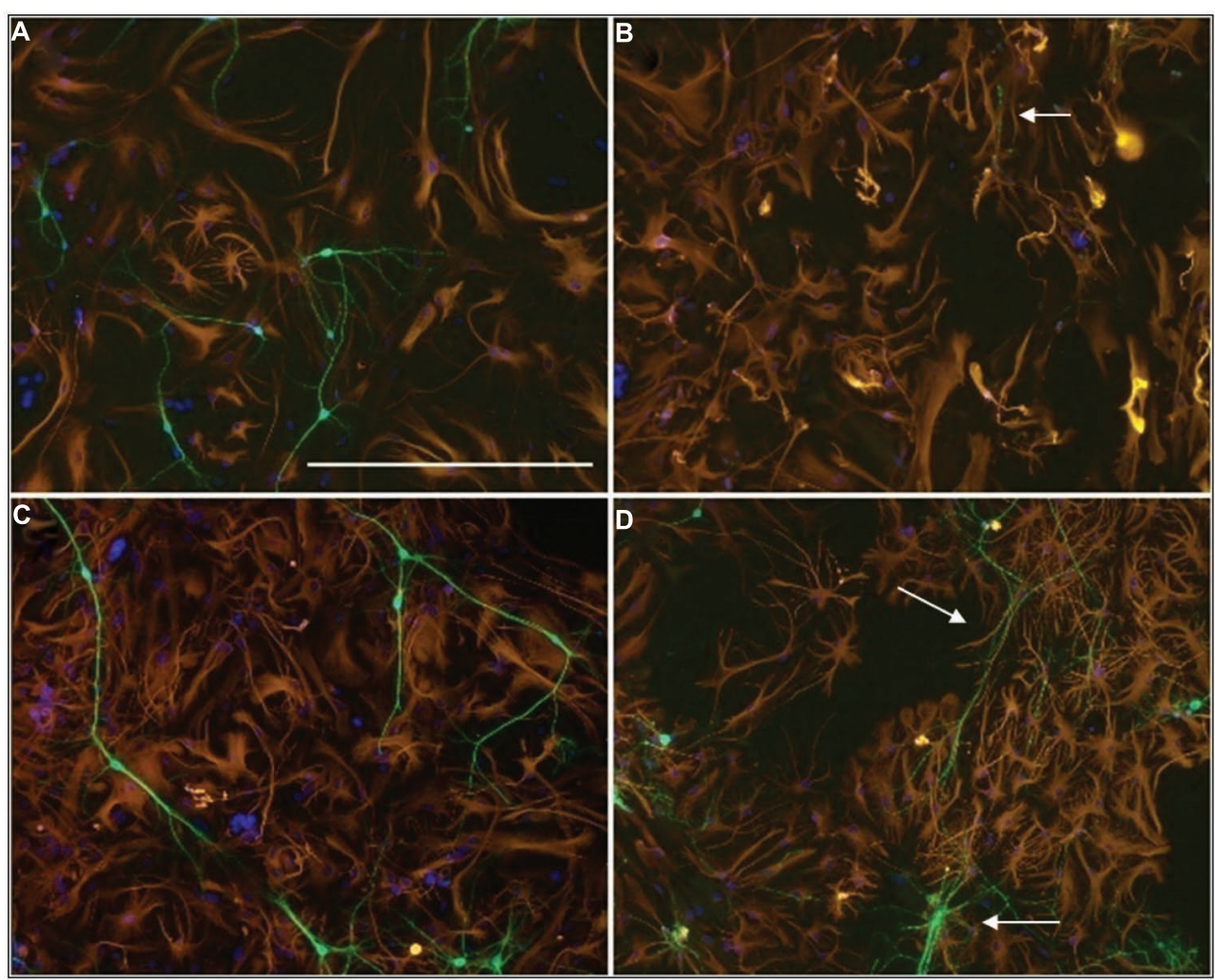

E

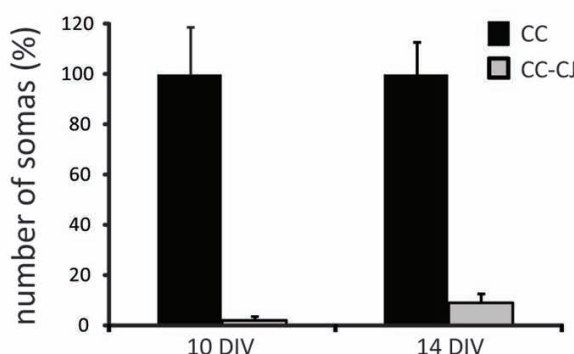

FIGURE 1 | Efficiency of cold jet on neuron-astrocyte co-cultures after 10 and 14 DIV. (A) Neuron-astrocyte co-culture at 10 DIV. (B) At 10 DIV, cold jet removed almost all neuronal cell bodies and only a few residual neurites were detected (white arrow), note that the astrocyte cell layer remained unchanged. (C) Co-culture after 14 DIV. (D) At 14 DIV, cold jet removed neurites but less efficiently than at 10 DIV. Shown is an example were many
$\mathbf{F}$

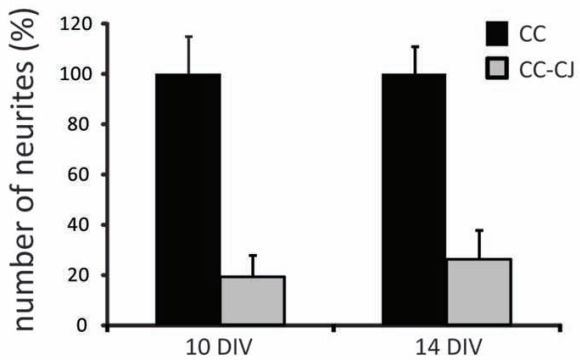

residual neurites are observed (white arrows), probably representing clusters of firmly attached neurons. Neuron-astrocyte co-cultures with or without treatment with cold jet, were stained for the neuronal marker MAP2 (green), the astrocyte marker GFAP (red), and nuclei [4',6-diamidino-2-phenylindole (DAPI), blue] to count the number of somas (E) and neurites (F). Numbers show average and SEM for three tissue culture wells. Scale bar $100 \mu \mathrm{M}$ (A).
Analysis of the microarrays served two purposes: (1) detection of neuron-induced gene expression in astrocytes and (2) determination of the cold jet efficiency on the mRNA level. In total, four arrays were used. For the first purpose, two CC-CJ samples were labeled with $\mathrm{Cy} 3$ and two AA-CJ samples were labeled with Cy5. Each CC-CJ sample was hybridized together with an AA-CJ sample to an array. For checking the cold jet procedure on the mRNA level, two co-culture (CC) samples were labeled with Cy5 fluorescence and hybridized on an array together with a CC-CJ sample (Cy3). The microarray dataset can be found at National Center for Biotechnology
Information Gene Expression Omnibus (NCBI GEO; accession number GSE52481).

Normalization of the raw data was performed, after feature extraction by Agilent software, by optimized local intensitydependent normalization (OLIN) and optimized scaled local intensity-dependent normalization (OSLIN; Futschik and Crompton, 2005) using the $\mathrm{R}$ project for statistical computing (version $6.0 ; \mathrm{R}$ project). Next, $M$ values were scaled so that upregulated probes were associated with positive values and downregulated probes with negative values. The following criteria were used for selection of regulated transcripts: (1) for each array, the mean 
A

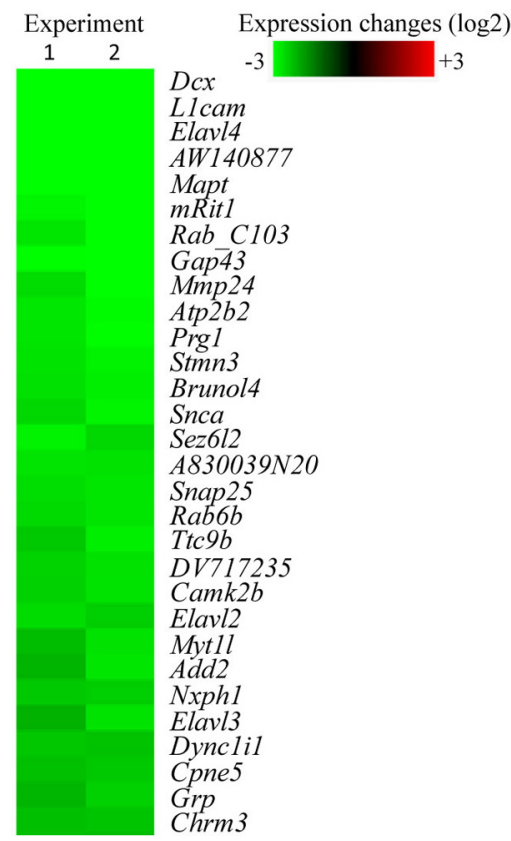

B

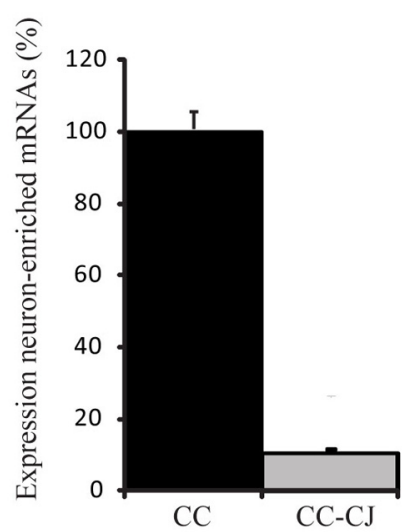

FIGURE 2 | Efficiency of cold jet to remove neuronal mRNA from co-cultures. (A) Samples from neuron-astrocyte co-cultures, with or without treatment with cold jet, were collected and mRNA levels for neuronal mRNAs were analyzed using microarray. Shown are 40 of the most down-regulated transcripts in co-culture as a result of the cold jet procedure. Each individual gene expression level was normalized (see Methods) and plotted on a Log2 scale, green representing decreased mRNA levels in CC-CJ samples compared to CC alone (B) Normalized fluorescence levels were compared and expressed as percentage of the levels present in co-culture samples. Error bars represent standard error of the mean (SEM). and standard deviation (SD) of the $M$ values of all probes was calculated, and only mRNAs with $M$ values equal or higher to the mean $+2 \times \mathrm{SD}$, or equal or lower to the mean $-2 \times \mathrm{SD}$ were selected, (2) $M$ values had to fulfill our criteria on both replicate arrays, (3) for mRNAs that were represented on the microarray with multiple probes, data were checked for consistency, and probes were only included in the analysis when replicate sequences gave comparable results, (4) mRNAs upregulated in the CC-CJ with AA-CJ comparison could represent left-over neuronal RNA when also strongly downregulated in the CC with CC-CJ comparison. Therefore, mRNAs upregulated in the CC-CJ with AA-CJ comparison where only included when downregulation in the CC with CC-CJ comparison was less than the mean $-2 \times$ SD. To assess consistency between technical and biological replicate samples, as well as between replicate probes, non-parametric correlations (using Spearman's rho) within nonnormalized red or green fluorescence samples were calculated. For technical/biological replicate samples, correlations were only performed on regulated probes as indicated by the cold jet-astrocyte alone arrays.

Thereafter, data were analyzed by TopGo (Alexa et al., 2006) using the R project for statistical computing. Overrepresented biological processes were identified by scoring statistical significance of predefined annotated groups based on the gene ontology (GO) database. Statistical significance of overrepresented predefined GO biological processes was scored using the Fisher exact test (Alexa et al., 2006).

\section{RESULTS}

\section{EFFECTIVENESS OF THE COLD JET METHOD ON NEURON-ASTROCYTE} CO-CULTURES

Neuronal development in hippocampal mixed neuron-glia cultures is a temporal process involving neurite outgrowth (1 DIV onward), and synapse formation from 4 DIV onward (Basarsky et al., 1994). Since attachment of neurons to the extracellular culture environment might vary with time, cold jet removal of neurons from neuron-astrocyte co-cultures was tested at 10 and 14 DIV. Quantification of the number of neuronal somas and neurites, indicated that cold jet removed over $98 \%$ of neuronal somas and $80 \%$ of neurites at 10 DIV, and was only slightly less efficient at 14 DIV (Figure 1). Cold jet removal of neuronal mRNAs was tested by microarray RNA profiling, which confirmed robust removal of neuronal mRNAs, e.g., Mapt, Gap43, Snap25, and Snca (Figure 2A). Neuron-enriched mRNAs are expected downregulated in coculture by cold jet, while being absent or low expressed in astrocytes. To determine the efficiency of the cold jet in removing these neuron-enriched mRNAs, their fluorescence levels in CC and CC-CJ was taken and the level in astrocytes substracted. The data indicated that the cold jet effectively removed approximately $90 \%$ of neuronal RNA (Figure 2B).

\section{GENE-EXPRESSION ANALYSIS OF NEURON-RESPONSIVE ASTROCYTE GENES}

Microarray analysis of CC-CJ versus AA-CJ samples, yielded 394 neuron-induced regulations of astrocyte mRNAs, of which 270 


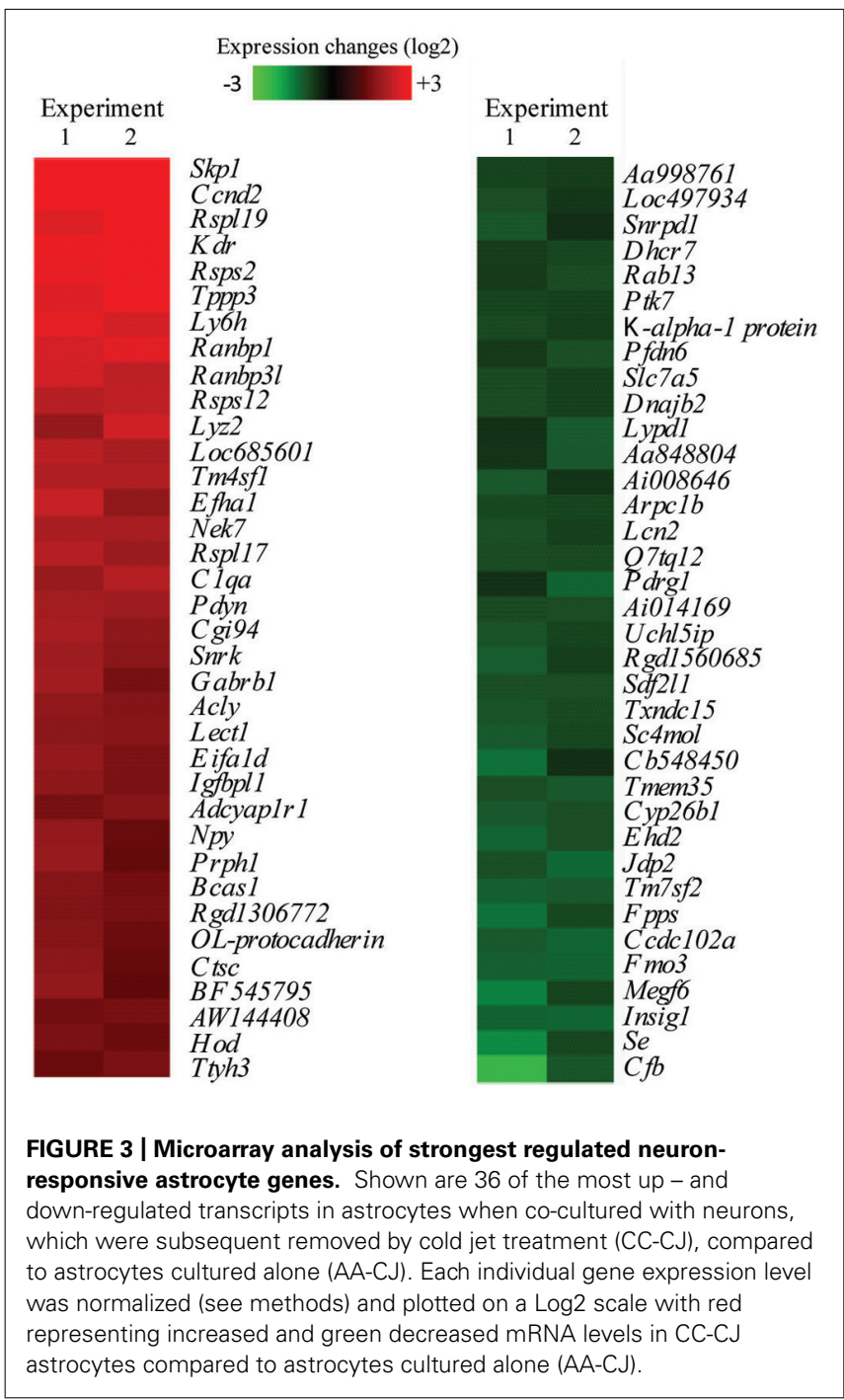

were upregulated and 124 downregulated. The 36 strongest upand down-regulated mRNAs are shown (Figure 3). The group of 270 mRNAs included many which are involved in astrocyteneuron interactions, such as cell adhesion (Itgb, Pcdh10, Cdh6, Ctnnal1, Glycam, L1cam, and TSP precursor 4), extracellular matrix (Cspg5 and Reck) and cytokine signaling (Megf6 and Lcn2). Several mRNAs involved in lipid synthesis were found down-regulated, including mRNAs involved in cholesterol synthesis (Dhcr7, Hmgcs, Sc4mol, Cyp26b, Tm7sf2, FPS, Sql, and Insig1).

To gain more insight into biological processes represented by the data set, we scored statistical significance of predefined functional genes groups, based on GO biological processes. From the complete list of regulated mRNAs (394), 10 significant overrepresented GO terms were identified (with $p<0.005$, see Table 1). Overrepresented GO terms included mainly metabolic processes, e.g., sterol and nutrient related processes. GO analysis of only up-regulated (Table 2) or only down-regulated (Table 3) gene groups showed most strikingly that mRNAs associated with "sterol metabolism" were mainly down-regulated, whereas "response to nutrient" processes were up-regulated.
Table 1 | Gene ontology-overrepresentation analysis for biological processes for all mRNAs (394) regulated in astrocytes when co-cultured with neurons.

\begin{tabular}{lll}
\hline GO ID & GO term & $\boldsymbol{p}$-Value \\
\hline GO:0016125 & Sterol metabolic process & $2.20 \mathrm{E}-10$ \\
GO:0006991 & Response to sterol depletion & $4.60 \mathrm{E}-07$ \\
GO:0031667 & Response to nutrient levels & 0.00019 \\
GO:0045332 & Phospholipid translocation & 0.00049 \\
GO:0015679 & Plasma membrane copper ion transport & 0.00082 \\
GO:0000245 & Spliceosome assembly & 0.00089 \\
GO:0001955 & Blood vessel maturation & 0.00288 \\
GO:0007218 & Neuropeptide signaling pathway & 0.00291 \\
GO:0006800 & Oxygen and reactive oxygen species & 0.00314 \\
& metabolism &
\end{tabular}

GO:0006968 Cellular defense response

0.00409

Table 2 | Gene ontology-overrepresentation analysis for biological processes for mRNAs (270) up-regulated in astrocytes when co-cultured with neurons.

\begin{tabular}{lll}
\hline Gene ontology ID & Gene ontology term & $\boldsymbol{p}$-Value \\
\hline GO:0007584 & Response to nutrient & 0.00013 \\
GO:0045332 & Phospholipid translocation & 0.00019 \\
GO:0015679 & Plasma membrane copper ion transport & 0.00032 \\
GO:0001955 & Blood vessel maturation & 0.00114 \\
GO:0008406 & Gonad development & 0.00132 \\
GO:0007218 & Neuropeptide signaling pathway & 0.00324 \\
GO:0006911 & Phagocytosis, engulfment & 0.00326 \\
GO:0007160 & Cell-matrix adhesion & 0.00475 \\
\hline
\end{tabular}

Table 3 | Gene ontology-overrepresentation analysis for biological processes for mRNAs (124) down-regulated in astrocytes when co-cultured with neurons.

\begin{tabular}{lll} 
Gene ontology ID & Gene ontology term & $\boldsymbol{p}$-Value \\
\hline GO:0016125 & Sterol metabolic process & $2.10 \mathrm{E}-11$ \\
GO:0006991 & Response to sterol depletion & $8.50 \mathrm{E}-09$ \\
GO:0000245 & Spliceosome assembly & 0.00055 \\
GO:0006481 & C-terminal protein methylation & 0.00342 \\
GO:0015819 & Lysine transport & 0.00342 \\
GO:0046498 & S-adenosylhomocysteine metabolic & 0.00382 \\
& process & \\
GO:0006776 & Vitamin A metabolic process & 0.0047 \\
\hline
\end{tabular}

To validate the microarray data, qPCR was performed on a selection of mRNAs that were regulated between astrocytes alone (AA-CJ) and astrocytes in co-culture (CC-CJ). qPCR confirmed the down-regulation of three mRNAs involved in lipid metabolism (Insig1, Hmgcs, and Dhcr7) and confirmed the up-regulation of 


\section{mRNA levels [log2(CC-CJ/AA-CJ)]}

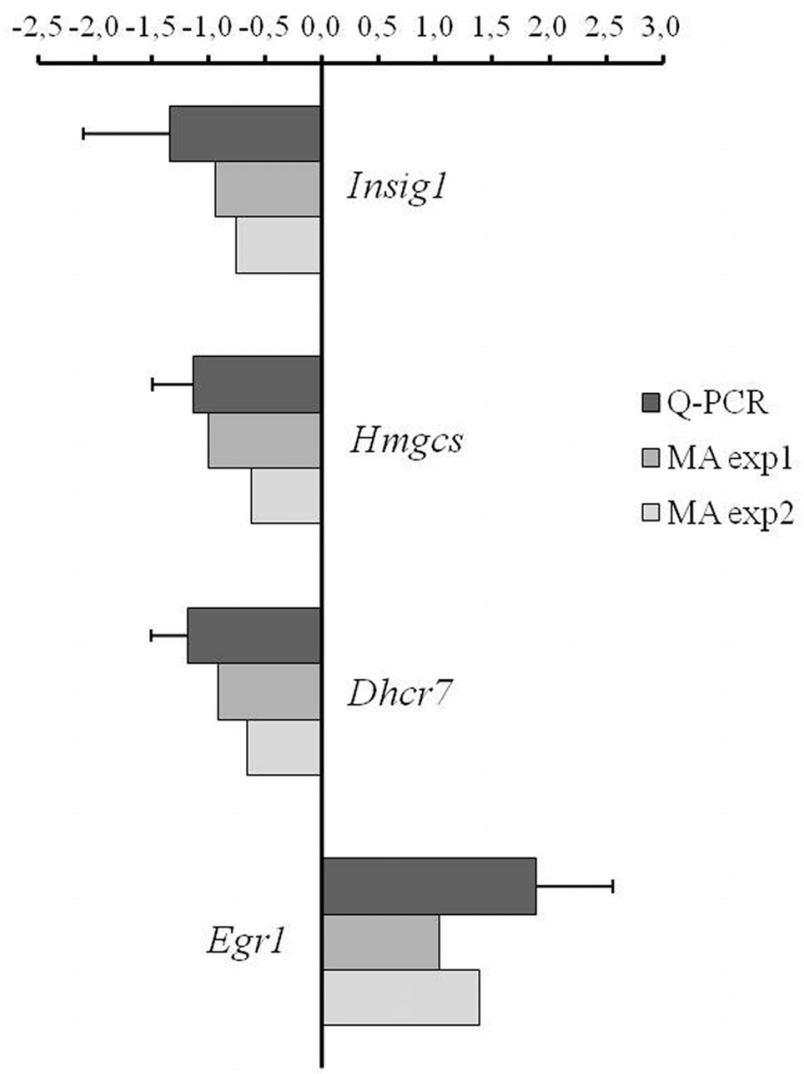

FIGURE 4 | Quantitative PCR analysis of neuron-responsive astrocytes genes. Transcript levels were measured using qPCR for a selected group of astrocyte-expressed mRNAs that according to microarray were neuron-responsive (in MA exp 1 and MA exp 2). The ratio between expression in CC-CJ vs. AA-CJ was calculated and plotted on a Log2 scale, e.g., expression of EGR1 was 3.7-fold higher in CC-CJ then in AA-CJ. Data represents the mean and SD of four samples.

the transcription factor Egr1 in astrocytes when co-cultured with neurons (Figure 4).

\section{DISCUSSION}

\section{COLD JET: METHOD FOR GENTLE SEPARATION OF NEURONS FROM CO-CULTURED ASTROCYTES}

Neuron-glia co-cultures are an established in vitro model to investigate the reciprocal interactions of these cell types that underlie neurite outgrowth, synapse formation, and synaptic transmission. Investigating the molecular changes in astrocytes that take place during interaction requires the efficient isolation of astrocytes from the co-culture in a manner that ensures cellular integrity. Here, we present cold jet as a fast and efficient isolation method of astrocytes from differentiating neuron-astrocyte co-cultures. We showed that at 10 and 14 DIV, when neurites and mature synapses have been formed (Basarsky et al., 1994), cold jet removed nearly all neuronal RNA in these co-cultures. This permits selective transcriptional analysis of only astrocytes in these co-cultures. Cold jet isolation of astrocytes from neuron-astrocyte co-cultures may also be suitable for analysis of proteins and lipids, since more than $90 \%$ of neuronal cell bodies and nearly as much neurites are removed. We conclude that cold jet offers important advantages over other techniques that are used for cell separation, such as FACS or immuno-panning. Unlike those other techniques, cold jet is fast (within $1 \mathrm{~min}$ ), easy to perform, done without addition of proteases, and conducted under ice-cold conditions to ensure no detriment to molecular integrity.

\section{IDENTIFICATION OF NEURON-RESPONSIVE ASTROCYTE GENES}

Through a developmental in vitro model, in which neurons in co-culture with astrocytes differentiate and form synapses, we set out to identify neuron-responsive astrocyte gene expression using microarray analysis. We found regulation of many genes involved in astrocyte biological processes that have been reported previously to affect neuronal differentiation and function, such as lipid metabolism (Camargo et al., 2009, 2012; Pfrieger and Ungerer, 2011), cell-ECM adhesion (Christopherson et al., 2005; Tournell et al., 2006), complement factor signaling (Stevens et al., 2007), cytokine signaling (Acarin et al., 2001), and synaptic transmission (Halassa and Haydon, 2010). It should be noted that most of the astrocyte mRNAs involved in cholesterol metabolism were down-regulated by neuronal presence, which is surprising considering previous findings illustrating the importance of astrocyte cholesterol for synapse maturation and neurite outgrowth (Mauch et al., 2001; Fan et al., 2002; Hayashi et al., 2004; Camargo et al., 2009). However, we did find down-regulation of Insig1, an important negative regulator of the lipogenic sterol regulatory element binding protein (SREBP) transcription factors, which therefore would be expected to lead to increased SREBP-mediated transcription of lipogenic enzymes, as found in other cell systems (Engelking et al., 2005). Our recent observation that SREBPs are also required for expression of lipogenic enzymes in astrocytes (Camargo et al., 2012), warrants further investigation of the control of Insig, SREBP, and downstream transcription in astrocytes due to contact with neurons. Taken together, our observations suggest that astrocyte-factors involved in these processes do not only influence neuronal differentiation but are furthermore induced in astrocytes by neuronal presence, underscoring the reciprocal nature of the neuron-glia interactions.

\section{ACKNOWLEDGMENTS}

Karen E. Carney was sponsored by the Erasmus Mundus ENC Network. Nutabi Camargo was on the Marie Curie ITN grant "Neuromics."

\section{REFERENCES}

Acarin, L., Gonzalez, B., and Castellano, B. (2001). Glial activation in the immature rat brain: implication of inflammatory transcription factors and cytokine expression. Prog. Brain Res. 132, 375-389. doi: 10.1016/S0079-6123(01)32089-7

Alexa, A., Rahnenfuhrer, J., and Lengauer, T. (2006). Improved scoring of functional groups from gene expression data by decorrelating GO graph structure. Bioinformatics 22, 1600-1607. doi: 10.1093/bioinformatics/btl140

Basarsky, T. A., Parpura, V., and Haydon, P. G. (1994). Hippocampal synaptogenesis in cell culture: developmental time course of synapse formation, calcium influx, and synaptic protein distribution. J. Neurosci. 14, 6402-6411.

Camargo, N., Brouwers, J. F., Loos, M., Gutmann, D. H., Smit, A. B., and Verheijen, M. H. (2012). High-fat diet ameliorates neurological deficits caused by 
defective astrocyte lipid metabolism. FASEB J. 26, 4302-4315. doi: 10.1096/fj.12205807

Camargo, N., Smit, A. B., and Verheijen, M. H. (2009). SREBPs: SREBP function in glia-neuron interactions. FEBS J. 276, 628-636. doi: 10.1111/j.17424658.2008.06808.x

Christopherson, K. S., Ullian, E. M., Stokes, C. C., Mullowney, C. E., Hell, J. W., Agah, A., et al. (2005). Thrombospondins are astrocyte-secreted proteins that promote CNS synaptogenesis. Cell 120, 421-433. doi: 10.1016/j.cell.2004. 12.020

Clarke, L. E., and Barres, B. A. (2013). Emerging roles of astrocytes in neural circuit development. Nat. Rev. Neurosci. 14, 311-321. doi: 10.1038/nrn3484

Engelking, L. J., Liang, G., Hammer, R. E., Takaishi, K., Kuriyama, H., Evers, B. M., et al. (2005). Schoenheimer effect explained - feedback regulation of cholesterol synthesis in mice mediated by Insig proteins. J. Clin. Invest. 115, 2489-2498. doi: 10.1172/JCI25614

Eroglu, C., and Barres, B. A. (2010). Regulation of synaptic connectivity by glia. Nature 468, 223-231. doi: 10.1038/nature09612

Fan, Q. W., Yu, W., Gong, J. S., Zou, K., Sawamura, N., Senda, T., et al. (2002). Cholesterol-dependent modulation of dendrite outgrowth and microtubule stability in cultured neurons. J. Neurochem. 80, 178-190. doi: 10.1046/j.00223042.2001.00686.x

Foo, L. C. (2013). Purification of rat and mouse astrocytes by immunopanning. Cold Spring Harb. Protoc. 2013, 421-432. doi: 10.1101/pdb.prot074211

Futschik, M. E., and Crompton, T. (2005). OLIN: optimized normalization, visualization and quality testing of two-channel microarray data. Bioinformatics 21 , 1724-1726. doi: 10.1093/bioinformatics/bti199

Giaume, C., Koulakoff, A., Roux, L., Holcman, D., and Rouach, N. (2010). Astroglial networks: a step further in neuroglial and gliovascular interactions. Nat. Rev. Neurosci. 11, 87-99. doi: 10.1038/nrn2757

Goritz, C., Mauch, D. H., and Pfrieger, F. W. (2005). Multiple mechanisms mediate cholesterol-induced synaptogenesis in a CNS neuron. Mol. Cell. Neurosci. 29, 190-201. doi: 10.1016/j.mcn.2005.02.006

Halassa, M. M., and Haydon, P. G. (2010). Integrated brain circuits: astrocytic networks modulate neuronal activity and behavior. Annu. Rev. Physiol. 72, 335355. doi: 10.1146/annurev-physiol-021909-135843

Hayashi, H., Campenot, R. B., Vance, D. E., and Vance, J. E. (2004). Glial lipoproteins stimulate axon growth of central nervous system neurons in compartmented cultures. J. Biol. Chem. 279, 14009-14015. doi: 10.1074/jbc.M3138 28200

Jarjour, A. A., Zhang, H., Bauer, N., Ffrench-Constant, C., and Williams, A. (2012). In vitro modeling of central nervous system myelination and remyelination. Glia 60, 1-12. doi: 10.1002/glia.21231

Jirsova, K., Sodaar, P., Mandys, V., and Bar, P. R. (1997). Cold jet: a method to obtain pure Schwann cell cultures without the need for cytotoxic, apoptosisinducing drug treatment. J. Neurosci. Methods 78, 133-137. doi: 10.1016/S01650270(97)00146-5

Lovatt, D., Sonnewald, U., Waagepetersen, H. S., Schousboe, A., He, W., Lin, J. H., et al. (2007). The transcriptome and metabolic gene signature of protoplasmic astrocytes in the adult murine cortex. J. Neurosci. 27, 12255-12266. doi: 10.1523/JNEUROSCI.3404-07.2007
Mauch, D. H., Nagler, K., Schumacher, S., Goritz, C., Muller, E. C., Otto, A., et al. (2001). CNS synaptogenesis promoted by glia-derived cholesterol. Science 294, 1354-1357. doi: 10.1126/science.294.5545.1354

Medina, J. M., and Tabernero, A. (2002). Astrocyte-synthesized oleic acid behaves as a neurotrophic factor for neurons. J. Physiol. Paris 96, 265-271. doi: 10.1016/S0928-4257(02)00015-3

Mendez, J. A., Lopez-Bayghen, E., Rojas, F., Hernandez, M. E., and Ortega, A. (2004). Glutamate regulates Oct-2 DNA-binding activity through alpha-amino-3hydroxy-5-methylisoxazole-4-propionate receptors in cultured chick Bergmann glia cells. J. Neurochem. 88, 835-843. doi: 10.1046/j.1471-4159.2003.02206.x

Pfrieger, F. W., and Ungerer, N. (2011). Cholesterol metabolism in neurons and astrocytes. Prog. Lipid Res. 50, 357-371. doi: 10.1016/j.plipres.2011.06.002

Stevens, B., Allen, N. J., Vazquez, L. E., Howell, G. R., Christopherson, K. S., Nouri, N., et al. (2007). The classical complement cascade mediates CNS synapse elimination. Cell 131, 1164-1178. doi: 10.1016/j.cell.2007.10.036

Thomson, C. E., McCulloch, M., Sorenson, A., Barnett, S. C., Seed, B. V., Griffiths, I. R., et al. (2008). Myelinated, synapsing cultures of murine spinal cord - validation as an in vitro model of the central nervous system. Eur. J. Neurosci. 28, 1518-1535. doi: 10.1111/j.1460-9568.2008.06415.x

Tournell, C. E., Bergstrom, R. A., and Ferreira, A. (2006). Progesteroneinduced agrin expression in astrocytes modulates glia-neuron interactions leading to synapse formation. Neuroscience 141, 1327-1338. doi 10.1016/j.neuroscience.2006.05.004

Ullian, E. M., Sapperstein, S. K., Christopherson, K. S., and Barres, B. A. (2001). Control of synapse number by glia. Science 291, 657-661. doi: 10.1126/science.291.5504.657

Verheijen, M. H., Camargo, N., Verdier, V., Nadra, K., De Preux Charles, A. S., Medard, J. J., et al. (2009). SCAP is required for timely and proper myelin membrane synthesis. Proc. Natl. Acad. Sci. U.S.A. 106, 21383-21388. doi: 10.1073/pnas.0905633106

Wierda, K. D., Toonen, R. F., De Wit, H., Brussaard, A. B., and Verhage, M. (2007). Interdependence of PKC-dependent and PKC-independent pathways for presynaptic plasticity. Neuron 54, 275-290. doi: 10.1016/j.neuron.2007.04.001

Conflict of Interest Statement: The authors declare that the research was conducted in the absence of any commercial or financial relationships that could be construed as a potential conflict of interest.

Received: 16 September 2013; accepted: 08 January 2014; published online: 30 January 2014.

Citation: Goudriaan A, Camargo N, Carney KE, Oliet SHR, Smit AB and Verheijen MHG (2014) Novel cell separation method for molecular analysis of neuron-astrocyte co-cultures. Front. Cell. Neurosci. 8:12. doi: 10.3389/fncel.2014.00012

This article was submitted to the journal Frontiers in Cellular Neuroscience.

Copyright ( $) 2014$ Goudriaan, Camargo, Carney, Oliet, Smit and Verheijen. This is an open-access article distributed under the terms of the Creative Commons Attribution License (CC BY). The use, distribution or reproduction in other forums is permitted, provided the original author(s) or licensor are credited and that the original publication in this journal is cited, in accordance with accepted academic practice. No use, distribution or reproduction is permitted which does not comply with these terms. 